\title{
Raising the bar
}

\author{
We are changing the way we evaluate papers to ensure timely dissemination of chemical biology \\ research.
}

Since the inaugural issue of Nature Chemical Biology, the editorial team has aimed to create a top-tier journal that serves an international readership and reflects the diversity of chemical biology research by identifying and publishing the highest-quality papers that we receive (Nat. Chem. Biol. 1, 3, 2005; Nat. Chem. Biol. 7, $649,2011)$. In parallel, we have endeavored to foster communication in the field by publishing papers that promote scientific discourse and by interacting directly with our readers, authors and referees to establish rigorous yet realistic standards for the community. These conversations have been critical in shaping our editorial processes as well as in defining the scope of the journal. This ongoing dialogue, in conjunction with increased competition for space in our pages, has prompted new changes in our editorial processes to place greater emphasis on providing rapid and decisive feedback for all potential authors and to promote timely dissemination of research to our readership and the chemical biology community at large.

Chemical biology as a scientific discipline is booming, with the increasing excitement, respect and resources available to the field reflected in the growth of the journal since its inception. For example, in the first 12 issues of the journal, we featured 63 original research reports; in the most recent 12 , this number rose to 121 . The journal's growth also derives from an editorial commitment made several years ago to better capture the full breadth of the field by increasing the volume of research papers published in our pages. In practice, this goal was achieved by promoting transparency in our decisions and by reviewing some papers that reported interesting findings but required significant development to be both biologically relevant and chemically rigorous. Though we believe these strategies were successful and have contributed to the increased profile of the journal and chemical biology as a discipline, we have exhausted our ability to expand the pages in the journal allotted to primary research papers for the present. In tandem, we have seen a persistent rise in the quality and quantity of manuscripts submitted to the journal; these combined factors mean that we are turning away increasing numbers of interesting reports.

These changes at Nature Chemical Biology have coincided with a time when the scientific community as a whole has voiced the opinion that peer review at many journals has become too onerous, involving too many rounds of revision that delay publication and burden both authors and referees. In conjunction with an internal review of our editorial strategies, this valuable community feedback has led us to make several fundamental changes to our processes. First and foremost, we are prioritizing our commitment to a timely peer-review and publication process, meaning that we aim to select papers not on the basis of their potential but rather on the data at hand. Further, we expect to gain a clear understanding of whether a paper is on track to publication in just one or two rounds of review. In practice, this means that some papers that might have been reviewed in the past are no longer being sent to external referees. It also means that if referees do not express high enthusiasm for the potential importance and technical quality of a study after the first round of review, it is unlikely that we will be able to invite the authors to revise the manuscript. Finally, to maximize the number of papers that we can publish, we are evaluating the content of each paper and providing tailored instructions for revision. This guidance is intended to eliminate redundancies within a paper that take up unnecessary space while retaining the clarity that is critical for our interdisciplinary readership.

These procedural changes will also affect our handling of appeals on papers that have been rejected without review for being preliminary or that have been rejected after review because the referees were not enthusiastic about the study. In the early days of the journal, we aimed to help interested authors revise and extend their manuscript before or after review, as chemical biology as a discipline, and thus the associated parameters for publishing, were in transition. Although it is true that a subset of recently rejected papers could still be successful if expanded, in practice these appeals will more consistently be turned down because the considerable time needed for extensive new experimentation can unnecessarily delay publication of work that may already be suitable for another venue.

Although these changes in our editorial processes will most likely be noted by the community, they do not entail a wholesale revision of our approach. Processes that have persistently been well received, particularly our willingness to communicate with authors before, during and after submission and review, will stand. We will also continue to carefully evaluate referee feedback on manuscripts, both to inform decisions on individual papers and to maintain consistent editorial standards across papers that are reviewed by distinct peer groups. Additionally, though we have long sought to protect our referees from spending time evaluating incomplete revisions, we will continue to seek referee advice on new data or substantive textual revisions, as we consider this practice critical to publishing sound and compelling scientific papers. However, we will strengthen our efforts to help authors navigate referee feedback and journal standards so that authors, referees and editors can more quickly determine whether a paper is ready for publication.

We believe these changes will help us to meet our overarching goal of providing a timely outlet for high-quality chemical biology research in the context of an increasingly competitive landscape. In addition, the changes should help papers that are not well placed with us move more rapidly to another outlet for publication. It has been exciting and inspiring to witness the rise of chemical biology and to develop a close relationship with this community of scientists. We look forward to working with you to ensure its continued success. 